\title{
Is a metonymy always a metonymy? A speculation on taxonomical aspects of propositional metonymy and situational metonymy
}

\author{
Nikolay Golovko ${ }^{1, *}$ \\ ${ }^{1}$ North Caucasus Federal University, Humanities Institute, 355009, 1 Pushkin street, Stavropol, Russia
}

\begin{abstract}
Research efforts in cognitive linguistics are frequently focused on the notion of metaphor, while the notion of metonymy, as well as its taxonomical aspects, is not always thoroughly researched. A study of the most recent research papers in the English language has shown that metonymy is often interpreted in a broad fashion, incorporating several different types of cognitive processes and phenomena, so that a scholar becomes unable to distinguish them from each other and / or is essentially forced to regard them as manifestations of the same phenomenon. A more taxonomically accurate approach is suggested, involving the use of the term "synecdoche" for affinity-related concept shifts and including a review of two subclasses of metonymy that have not yet received a considerable amount of attention - propositional metonymy and situational metonymy.
\end{abstract}

\section{Introduction}

Research efforts in the field of cognitive linguistics are frequently concentrated on questions and problems that are related to essence, functions and representations of metaphors, as well as to natural language processing operations on them. However, other referential means are still attracting less attention, even though there definitely is an amount of research in these adjacent areas. One of such means is the phenomenon of metonymy. Historically, it has been analyzed and reviewed by certain authors, of whom the following can be singled out: Lakoff (1987), Kövecses and Radden (1998), Panther and Thornburg (1999), Warren (1999), Mendoza Ibáñez and Campo (2001), Barcelona (2003), Deignan (2005), Denroche (2015). In the recent years, comprehensive theoretical research on metonymy has been relatively rare, conditioning several problems that will be presented further in the paper.

During our review of recent proceedings and findings dedicated to cognitive linguistics, studies on metaphors and other associated subjects, we have discovered references to several theoretical developments on the notion of metonymy that are still not explored well. In particular, we wanted to focus our attention on two exotic subclasses of metonymy suggested by Mendoza Ibáñez and Campo (2001), i.e. propositional metonymy and situational metonymy. We could not find any recent substantial research on these two categories, while we believe that they require rather serious consideration in terms of whether they should indeed be regarded as manifestations of metonymy.

Whereas the metaphor is receiving a more or less uniform approach to its features and definition, and its researchers appear to generally agree on the idea that it represents transfer of qualities between two objects based on similarity between them, metonymy often lacks accurate interpretation. As far as studies on it are less frequent, the unrefined condition of this aspect of theory is understandable. Still, it is important to clarify definitions, because our analysis suggests that various authors have been expanding the notion of metonymy towards concepts and phenomena that do not belong to it. The partially indefinite status of metonymy is the first problem to be explored.

The second problem consists in limited research available on two subclasses of metonymy, propositional metonymy and situational metonymy. It is our belief that existing suggestions to distinguish these categories of metonymical transfer are not entirely accurate, and we find it possible to claim that these concepts were grounded by an expansive interpretation of metonymy, allowing the respective authors to link this notion with phenomena that lie beyond its limits. In particular, the concepts appear to stem from understanding metonymy as "part instead of whole" type of relationship, while, for example, the Russian linguistic and literary tradition (that we belong to) separates such references from the notion of metonymy and singles them out as an independent device known as synecdoche.

The statement of these two problems leads us to formulation of certain research questions, listed in the following part of this article.

The initial research question is whether the scholars who have been studying the language means of metonymy during the recent years are establishing their research on a reliable interpretation of this term. In order to be able to respond to this question, we will need to

\footnotetext{
Corresponding author: nvgolovko@inbox.ru
} 
review definitions of metonymy that are found in actual research articles and analyze them critically, as well as suggest a refined interpretation in case of necessity. The secondary question is related to two aforementioned subclasses of metonymy; we intend to check whether propositional metonymy and situational metonymy are in harmony with the most accurate definition of metonymy as a linguistic, communicative and cognitive phenomenon that we will be able to provide.

The choice of research questions determines the purpose of this study, as well as materials and methods that we will need to use.

The ultimate purpose of this study is to either confirm or suggest to revise the status of propositional metonymy and situational metonymy on the basis of analyzing and refining the definition of metonymy itself. As far as materials and methods are concerned, they can be found in the next section of the paper.

\section{Materials and Methods}

Achievement of the purpose of this study may be possible through the following procedures:

1) We would collect open-access research papers on metonymy that were published in the recent 3 years (since 2016) and indexed in any of the Web of Science core databases. We would then review the theoretical approaches to metonymy that are found in these papers, analyze them and compare against the traditional Russian academic and scientific interpretation that we are used to rely on. We would also try to formulate an improved definition of the term, based on the results of this analysis and comparison.

2) We would cross-reference the refined definition of metonymy with the concepts of propositional metonymy and situational metonymy, that we are particularly interested in. Through resulting speculation and reflection on accuracy of these terms, as they are suggested by Mendoza Ibáñez and Campo (2001), we would work towards a critical evaluation of these concepts, and of the degree of their relationship to metonymy in particular.

\section{Discussion}

\subsection{The definition of metonymy in recent research}

In accord with the criteria that we have defined in the previous section of the paper, 10 research articles have been extracted and analyzed.

Arijana Krišković in Metonymic and non-metonymic use of names for branches of medical science in English and Croatian: a cognitive linguistic analysis (2016) relies on Lakoff (1987) and Barcelona (2003) in their interpretation of metonymy, stating that it is a cognitive mechanism in which an element of one domain is interpreted in terms of another domain, included in the same shared experiential domain [1].
Lewis Bott, Alice Rees and Steven Frisson in The Time Course of Familiar Metonymy (2016) define metonymy as "a form of figurative language in which people refer to an entity (e.g., the institution governing a hospital) by a salient property of the entity (e.g., a hospital)" [2].

Aseel Zibin's On the production of metaphors and metonymies by Jordanian EFL learners: acquisition and implications (2016) makes use of a definition from Deignan (2005), suggesting that metonymy should be regarded as "indirect reference, or reference shift, in which a linguistic sign refers not [only] to its default concept $\mathrm{A}$, but to another concept $\mathrm{B}$, within a single cognitive domain" [3].

In Maria M. Piñango's Metonymy as referential dependency: psycholinguistic and neurolinguistic arguments for a unified linguistic treatment (2016), it is claimed that "metonymy appears as a general type of semantic relation that allows a given term to be used to refer not to its original denotation, but to another denotation which is conversationally informative and one with which it holds some kind of functional correspondence" [4].

Sara Quintero Ramírez in Metonymy as a cohesion tool in sports newspaper articles (2017) is quoting Denroche (2015), building their understanding of metonymy on the idea that it is "the highlighting of relatedness, usually part-whole, between closely-related concepts, things and signifiers" [5].

Lidija Orčić and Sabina Zenejlagić in Metonymic and metaphoric extensions of the expression "keep an eye on somebody/something" (2018) are also relying on Lakoff's ideas. In particular, the researchers are using the phrase which states that metonymy "allows us to use one entity to stand for another" [6]. They also add the idea from Warren (1999), emphasizing the notion of contiguity as the core aspect of metonymy.

El Mustapha Lemghari in Le nom propre en lecture qualitative : de la métonymie à la métaphtonymie (2018) is concentrating on differences between qualitative metonymy and quantitative metonymy. They refer to Gary-Prieur (1994) and seem to interpret the notion of metonymy as the name shift between entities of different orders (primary / secondary) [7].

Jo Van Herwegen and Gabriella Rundblad in $A$ cross-sectional and longitudinal study of novel metaphor and metonymy comprehension in children, adolescents, and adults with autism spectrum disorder (2018) do not appear to provide a definition of metonymy; however, they distinguish "object-user" metonyms and "synecdoche metonyms" as separate notions [8].

Ana Belén Cabrejas-Peñuelas in Metonymy in Spanish and American parliamentary speeches: Obama's State of the Union address versus Rajoy's State of the Nation address (2018) is relying on definition from Kövecses \& Radden (1998), in accord with which metonymy is a "cognitive process in which one conceptual entity, the vehicle [or source], provides mental access to another conceptual entity, the target, within the same domain, or I(dealized) C(ognitive) M(odel)" [9]. 
Jeannette Littlemore and Caroline Tagg in Metonymy and Text Messaging: A Framework for Understanding Creative Uses of Metonymy (2018) are interpreting metonymy as "the use of one entity, process or event to refer to another related entity, process or event" [10].

Thus, there appears to be a consensus that concepts undergoing metonymic modifications are related to each other in a certain way. However, the essence of this relationship is rarely specified, and the authors seem to intentionally use broad phrasing in order to embrace the widest scope of phenomena possible. As a result, a considerable number of different relationships, such as "part-whole", "producer-product", "object-user", "effectcause" etc., becomes associated with metonymy.

The Russian linguistic and literary tradition interprets metonymy as a reference based on contiguity. On the wording level, this representation of the term is close to Warren's idea quoted in (Orčić 2018). Nonetheless, we find it possible to claim that the understanding of "contiguity" may differ across various scientific traditions. The definitions of metonymy that we found in publications in the English language appear to regard contiguity as the condition of being closely related on any basis. Lakoff's fundamental idea of entities belonging to the same cognitive domain can be used as an example: essentially, it represents another way to speak about contiguity. Otherwise saying, any subjects and / or objects that are somehow adjacent to each other may be called contiguous and thus may be subject to a metonymical shift. On the other hand, for a Russian researcher the idea of "contiguity" is more or less limited to that of spatial connection, where entities are located immediately next to each other; a typical example would be The entire room laughed, where the chamber is used as a reference to people occupying it.

Another aspect of this comparison between the Russian tradition and recent interpretations from the research in the English language would be the special status of "part-whole" relationship observed in the latter. We are used to the idea of singling out the referential means known as synecdoche for this certain variant of connection between subjects, objects or phenomena. As seen in (Herwegen \& Rundblad 2018), the notion of synecdoche is not alien to English-speaking scholars. At the same time, Denroche's quotation from (Ramirez 2017) clearly links this kind of relationship with metonymy.

There is, undoubtedly, an idea that synecdoche is a part of metonymical shifts and should thus be regarded as a subclass of metonymy. However, we would like to agree with Ken-ichi Seto, who argued in Distinguishing metonymy from synecdoche (1999) that it is an independent device based on a different type of reference [11]. The researcher emphasized the contrast between spatial / temporal nature of metonymic transfers and categorial nature of synecdoche; we believe that this consideration is noteworthy.

The core matter of this situation is not about mere terminological or taxonomical nuances, as it may seem upon the first glance. We are dealing with different types of relationships between objects or phenomena, and consequently, with different cognitive models, operations, and correlations. Therefore, whenever these relationships are studied within the context of cognitive linguistics, it is important to separate them: contiguity, or the condition of being adjacent in time and / or space, is not equal to "whole-part" association, as the former may imply a coincidental nature of the connection between them, whereas the latter always conveys the idea of "affinity", a sort of internal cohesion.

In a research paper on metonymy, there was the following example: Bush sent troops to Iraq. If we compare it to similar phrase Washington (D.C.) sent troops to Iraq, we will observe a certain difference: the second phrase is more abstract, referring to "people who are located in Washington" by the name of the capital of the United States, while the first phrase is more concrete, in part through the use of reference to George W. Bush, who is a human and consequently a part of the American nation. We find it possible to claim that Washington sent troops to Iraq contains implication "the U.S. government decided so", but Bush sent troops to Iraq may imply "the American people decided so".

The previous example that we have provided, The entire room laughed, is also a good sample to illustrate this idea. The individuals who are located in the room are not a part of it, so they do not inherit any of its qualities, as they do not have any affinity or kinship with it. Thus, their experience of laughing is not derived from the room or conditioned by it; the audience would have laughed in exactly the same manner in any other room. However, if we modify one of the reviewed authors' examples and say The suits would just laugh at this, such a statement would emphasize the non-coincidental and regular essence of the event, implying that the reaction of laughter is related to the formal style of clothing (which is in turn derived from the people's social roles and positions).

In this respect, we suggest to limit the notion of metonymy to spatial and / or temporal transfers, as well as other relationships that do not involve a direct causal connection between objects or phenomena. As far as the transfers based upon conditioned relationships, and the "part-whole" relationships in particular, are concerned, we find it possible to suggest to distinguish them from the notion of metonymy, as well as persistently use the term "synecdoche", because this variant of relationship may be associated with different cognitive mechanisms and should thus be studied separately. Other kinds of relationship might also be reviewed in this respect: for example, the "effect-cause" relationship implies derivation rather than temporal contiguity.

In addition, we find it worthy of emphasizing that in order to remain within the region of cognitive linguistics, one should add language-related references to their definitions. A definition stating that metonymy is a process where one entity is used instead of another is, in our opinion, more suitable for psychology or other related disciplines; in terms of linguistics, it might have been more accurate to state that name of one entity is used instead of another (the definitions provided by Deignan or Piñango are good examples). 


\subsection{The notions of propositional metonymy and situational metonymy}

It appears to us that the notions of propositional metonymy and situational metonymy were suggested by Ruiz de Mendoza Ibáñez and Otal Campo in High-level metonymy and linguistic structure (2001). Describing the first of these terms, the authors state that it applies when "a concept stands for another in a domain-internal relationship" and provide an example of "face for person" relationship, with 'face' being a subdomain of 'person'. As far as the second term is concerned, they characterize it through the situations where "a highly striking or otherwise significant element of a specific situation is used to stand for the larger event of which it forms part" [12]. It seems that the authors designed these notions to complement the taxonomy of referential, predicative and illocutionary metonymy suggested by other scholars.

In these statements, the idea of broader interpretation of metonymy is implemented; it is evident that both the example of propositional metonymy and the definition of situational metonymy are formulated with the "wholepart" relationship in mind. It would have been inappropriate to say that this approach is incorrect; it only follows a theoretical concept that was positively accepted and is still broadly used in the linguistic research in the English language. However, in the light of our previously described considerations, we find it possible to argue that first of all, these notions should be associated with synecdoche rather than with metonymy, as, once again, the relationship between the whole and its parts is different from the idea of contiguity conveyed by metonymical transfers.

In their example 2a, Mendoza Ibáñez and Campo suggest the following illustration of propositional metonymy: Tired faces all of them, some old, some young. Undoubtedly, an individual's facial expression is conditioned by their emotional and physical state and derived from it, so this state is transmitted through an object that constitutes a part of said individual rather than through objects that are merely adjacent to them. In our opinion, this situation indicates that the broad interpretation of metonymy might not always be beneficial for researches in cognitive linguistics; at least, the relationship between $\mathrm{A}$ and $\mathrm{B}$ where both $\mathrm{A}$ and $\mathrm{B}$ belong to the same domain should be distinguished from the relationship where $\mathrm{A}$ is a subdomain of $\mathrm{B}$.

The second part of the same illustration, Blufton smiled with pleasure and kept searching for their faces (example 2b), is to an extent different. We believe that it might need to be reconsidered in terms of whether it is indeed a transfer of any kind. This statement can be interpreted in a straightforward manner: the subject might have indeed been looking for faces, so it is somewhat open to argument whether 'face' is used here as an actual reference to an individual or as only a direct object of the person's mental activity.

For the notion of situational metonymy, the authors give an example of The poor dog left with its tail between its legs and suggest that a fragment of the scene (the animal moving away in a certain manner) is expanded into a larger picture where the dog undergoes certain negative experience resulting in this characteristic behavior. We personally regard this example, as well as the suggested notion itself, as dubious.

On one hand, there is no doubt in relation to the fact that language is tightly linked with thought and with ways of thinking. This enables the authors to define propositional metonymy via the notion of concept ("one concept stands for another"), and thus the idea of situational metonymy becomes a more or less simple expansion of the term: instead of "concept", a broader "situation" is manifested. Mendoza Ibáñez and Campo are by no means alone in their interpretation of metonymy: in the definitions we have previously listed, many scholars are regarding metonymy as a cognitive process rather than a linguistic phenomenon, and consequently, they represent it more immediately, as a concept shift rather than a name shift. It would have been challenging to argue against the approach of going deeper, right into the cognitive processes, beneath the surface of linguistic reflection of these processes; however, the linguistic part of the equation should not be put aside entirely.

On the other hand, whereas a concept is a result of a cognitive process (such as generalization of available facts about the environment and selection of characteristic features that would separate the concept from any other and constitute its content), is usually performed via language and is associated with a verbal identifier, a situation is not necessarily of such a "conceptual" nature. It is often a single concrete representative of a class rather than the class itself. Simply put, there is a fundamental difference between "face" as a concept and a certain given face as a representative of this concept: human thinking accumulates and amasses many examples of individual faces and generalizes this information through cognitive processes into a concept. Likewise, a situation we observe and perceive (the dog was punished and left with its tail between its legs) is a single event rather than a result of a cognitive process (even though there definitely exist cognitive activities related to its perception and representation in memory and conscience).

In addition, whenever linguistic terms, such as "metonymy", are used, they should be linked with language-related phenomena. Otherwise, as we stated above, there is a risk of leaving the "Linguistics" part of "Cognitive Linguistics" behind. We experience difficulties when trying to see a linguistic phenomenon in using a part of a situation to refer to the situation itself; it is, beyond doubt, a cognitive phenomenon, but the application of linguistic terms to it looks far-fetched to an extent. The same objection might be suggested against definitions where metonymy is interpreted as the use of one event instead of another: substitutions between events and their parts, in our opinion, is not exactly the same compared to using the verbal identifier of one event in order to denote another. This area requires additional consideration. 


\section{Conclusions}

The following conclusions can be derived:

1) In the current research in the English language, the concept of metonymy is interpreted broadly and embraces several different cognitive relationships between objects or phenomena. For example, the idea of contiguity (the state of being adjacent in time or space) is mixed with the notion of affinity (the state of providing or receiving inherited, shared or otherwise conditioned properties). It might be taxonomically and academically beneficial to separate these variants and single the affinity-based relationships out under the name of synecdoche. In case of limiting the scope of metonymy to spatial and temporal shifts, the status of some other relationships, such as "object-user" or "effect-cause", would require further analysis and probable reconsideration.

2) The notions of propositional metonymy and situational metonymy, suggested by Mendoza Ibáñez and Campo, are completely based upon the relationship of affinity ("whole-part") by their definitions, while the adjacent notions of referential, predicative and illocutionary metonymy do not presuppose such limitations and may imply other types of relationships. It may be advised to separate them taxonomically (i.e. use terms such as propositional synecdoche instead).

3) The notion of situational metonymy involves phenomena that appear to lie beyond the scope of linguistics: it leaves the area of relationships between language signs and their meanings or references, and it enters the area of relationships between mental images and / or observed events taking place in the environment.
Even though a verbal description of situations and their fragments can be regarded as a complex language sign, the idea of using linguistic terms, such as "metonymy" or "synecdoche", in this context might be regarded as objectionable, so future research in this area may be suggested.

\section{References}

1. A. Krišković, FLUMINENSIA, 28, 107-121 (2016)

2. L. Bott, A. Rees, S. Frisson, J. Exp. Psych., 42 (7), 1160-1170 (2016)

3. A. Zibin, Top. Ling. 17 (2), 41-58 (2016)

4. M. Piñango, M. Zhang, E. Foster-Hanson, M. Negishi, Ch. Lacadie, R. T. Constable, Cog. S. 41 (Suppl. 2), 351-378 (2017)

5. S. Quintero Ramírez, ELUA, 31, 269-284 (2017)

6. L. Orčić, S. Zenejlagić, FOLIA, 20, 87-103 (2018)

7. El M. Lemghari, SHS Web of Conferences, 46, 12004 (2018)

8. J. Van Herwegen, G. Rundblad, Front. Psychol., 9, 945 (2018)

9. A. Belén Cabrejas-Peñuelas, CLR, vol. XIX, 45-69 (2018)

10. J. Littlemore, C. Tagg, App. Lin., 39 (4), 481-507 (2018)

11. K. Seto, Metonymy in Language and Thought, 91121 (1999)

12. R. de Mendoza Ibáñez, O. Campo, Sincronia, 6/20 (2001) 